Kardiologe 2021 · 15:480-494

https://doi.org/10.1007/s12181-021-00499-0

Angenommen: 23. Juli 2021

Online publiziert: 13 . September 2021

(c) Deutsche Gesellschaft für Kardiologie - Herzund Kreislaufforschung e.V. Published by Springer Medizin Verlag $\mathrm{GmbH}$, ein Teil von Springer Nature - all rights reserved 2021

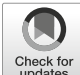

\section{Curriculum Kardiovaskuläre Schlafmedizin}

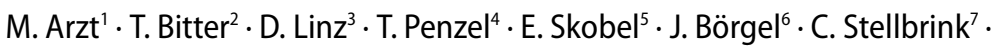 \\ O. Oldenburg ${ }^{8}$ \\ ' Klinik und Poliklinik für Innere Medizin II, Universitätsklinikum Regensburg, Regensburg, Deutschland \\ ${ }^{2}$ Städtisches Klinikum Braunschweig, Braunschweig, Deutschland \\ ${ }^{3}$ Universitätsklinikum des Saarlandes, Homburg/Saar, Deutschland \\ ${ }^{4}$ Charité Campus Mitte, Charité - Universitätsmedizin Berlin, Berlin, Deutschland \\ ${ }^{5}$ MVZ Luisenhospital, Aachen, Deutschland \\ ${ }^{6}$ St. Barbara-Klinik Hamm-Heessen, Hamm, Deutschland \\ ${ }^{7}$ Städt. Klinikum Bielefeld-Mitte, Bielefeld, Deutschland \\ ${ }^{8}$ Clemenshospital, Ludgerus-Kliniken Münster, Münster, Deutschland
}

Zusammenfassung

Schlafbezogener Atmungsstörungen sind bei kardiovaskulär erkrankten Patienten mit z. B. schwer einstellbarer arterieller Hypertonie, koronarer Herzerkrankung, Herzklappenerkrankungen, Herzrhythmusstörungen oder Herzinsuffizienz sehr häufig und mit einer schlechteren Prognose assoziiert. Die Therapie schlafbezogener Atmungsstörungen kann die Behandlung von Herzkreislauferkrankungen aufgrund ihrer Effekte auf den arteriellen Blutdruck und die Lebensqualität bei ausgewählten Patienten sinnvoll ergänzen. Umso wichtiger ist eine integrative kardiologische und schlafmedizinische Versorgung dieser Patienten. Zweck des Curriculums ist daher die Beschreibung eines Qualifizierungsprozesses, dessen Ziel die Erlangung der Zusatzqualifikation Kardiovaskuläre Schlafmedizin ist. Damit soll die Qualifizierung in diesem spezialisierten Bereich verbessert werden, sodass sie über das Maß der allgemeinen Facharztweiterbildung in der Kardiologie hinausgeht. Das Curriculum hat einen dreistufigen Aufbau. Stufe 1 vermittelt systematisch Grundkenntnisse und -fähigkeiten entsprechend den Qualifizierungsinhalten des Curriculums Kardiologie, Stufe 2 vermittelt aufbauend Kenntnisse und Fähigkeiten u. a. für die selbstständige Durchführung der Polygraphie und Stufe 3 u. a. für die selbständige Durchführung der Polysomnographie.

\section{Schlüsselwörter}

Obstruktive Schlafapnoe · Polygraphie · Polysomnographie · Qualität · Qualifizierung
Der Verlag veröffentlicht die Beiträge in der von den Autor*innen gewählten Genderform. Die Verwendung einer angemessenen gendergerechten Sprache, um Menschen in ihrer Vielfalt wertschätzend anzusprechen, wird begrüßt.

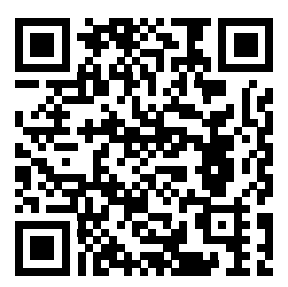

QR-Code scannen \& Beitrag online lesen

\section{Präambel}

Die Kardiologie hat sich, getragen durch die enormen Fortschritte im theoretischen Wissen und in den methodischen Möglichkeiten, in allen Facetten des Schwerpunkts erheblich weiterentwickelt [8]. Diese Entwicklung spiegelt sich auch in einer zunehmenden Spezialisierung von Kardiologen innerhalb des Schwerpunkts Kardiologie wider. Die Akademie Aus-, Weiter- und Fortbildung der Deutschen Gesellschaft für Kardiologie - Herz- und Kreislaufforschung e. V. (DGK) trägt dieser Entwicklung Rechnung und hat unter Mitwirkung von
Vertretern der jeweiligen Arbeitsgruppen Curricula für die Erlangung von Zusatzqualifikationen innerhalb des Schwerpunkts Kardiologie entwickelt. Diese Curricula sollen eine weitere Vertiefung von kardiologischem Wissen und technischen Fertigkeiten in definierten Subdisziplinen des Fachgebiets erleichtern. Sie bauen also einerseits auf den Inhalten der (Muster-)Weiterbildungsordnung im Schwerpunkt Kardiologie auf, gehen aber anderseits über das in der Weiterbildungsordnung für den Schwerpunkt Kardiologie geforderte theoretische Wissen und die technischen Fertigkeiten hinaus. 
Für diese Curricula wurde der Begriff Zusatzqualifikation gewählt, um diese zusätzliche Qualifizierung unter dem Dach der wissenschaftlichen Fachgesellschaft von der Regelweiterbildung nach der (Muster-)Weiterbildungsordnung der Bundesärztekammer sowie den Bestimmungen in der Umsetzung der jeweiligen zuständigen regionalen Ärztekammer zu unterscheiden. Gleichwohl haben die vorgestellten Curricula inhaltlich den Charakter einer über den Facharzt für Innere Medizin und Kardiologie bzw. Facharzt für Innere Medizin mit Schwerpunkt Kardiologie hinausgehenden Zusatzqualifikation und sollen aus Sicht der DGK auch eine solche Wirkung in der kardiologischen Versorgungslandschaft entfalten.

Im nachstehenden Text werden Personenbezeichnungen einheitlich und neutral für alle Geschlechter verwendet.

\section{Inhaltsverzeichnis}

1. Einleitung

2. Ziele

3. Syllabus

4. Durchführung der Qualifizierung

5. Anforderungen an den Programmkandidaten

6. Anforderungen an die Stätte der Zusatzqualifikation

7. Anforderungen an den (stellv.) Leiter der Zusatzqualifikation

8. Evaluierung

9. Übergangsregelung

10. Rezertifizierung

11. Qualitätskontrolle

12. Übersicht der erforderlichen Voraussetzungen

Literatur

\section{Einleitung}

Schlafstörungen und insbesondere schlafbezogene Atmungsstörungen umschreiben in der Kardiologie zunehmend beachtete Komorbiditäten. Schlafbezogene Atmungsstörungen treten per definitionem ausschließlich oder primär im Schlaf auf. Sie wirken störend auf den Schlaf und beeinträchtigen damit seine Erholungsfunktion [10]. Schlafbezogene Atmungsstörungen sind charakterisiert durch ein wiederkehrendes Auftreten von Apnoe- und
Hypopnoeereignissen mit Sauerstoffentsättigungen, Weckreaktionen und Aktivierungen des autonomen Nervensystems. Bei Patienten mit Herz-Kreislauf-Erkrankungen finden sich im Wesentlichen die obstruktive Schlafapnoe (OSA), die zentrale Schlafapnoe (ZSA) mit bzw. ohne Cheyne-Stokes-Atmungsmuster (CSA) oder die gemischtförmige Schlafapnoe [10].

Die Prävalenz von schlafbezogenen Atmungsstörungen ist abhängig von der kardialen und nichtkardialen Grunderkrankung, Geschlecht, Alter und im Falle der OSA vom Body-Mass-Index [1, 10]. Während die Prävalenz schlafbezogener Atmungsstörungen in der Allgemeinbevölkerung je nach Geschlecht und Alter zwischen 3 und $17 \%$ liegt, finden sich bei kardiovaskulär erkrankten Patienten mit koronarer Herzerkrankung, arterieller oder pulmonaler Hypertonie, Herzklappenerkrankungen, Herzrhythmusstörungen oder auch der Herzinsuffizienz Prävalenzen der relevanten schlafbezogenen Atmungsstörung von etwa 40-45\% [10].

Neben dieser hohen Prävalenz sind schlafbezogene Atmungsstörungen jedoch auch mit der Entstehung verschiedener kardiovaskulärer Erkrankungen assoziiert [4-7] bzw. unabhängig mit einer Verschlechterung der kardialen Funktion und Prognose verbunden $[2,3,9,11,12$, 16].

Umso wichtiger erscheint es, eine integrative kardiologische und schlafmedizinische Versorgung dieser Patienten zu gewährleisten. Da dies zum jetzigen Zeitpunkt weder durch den nicht auf schlafmedizinische Fragestellungen spezialisierten Kardiologen noch durch den nicht im Gebiet der Kardiologie erfahrenen Schlafmediziner gewährleistet werden kann, obliegt dem Curriculum Kardiovaskuläre Schlafmedizin der Anspruch, diese Lücke zu füllen und schlafmedizinisch interessierte Kardiologen sowohl mit einer entsprechenden Kompetenz zur Diagnostik und Therapie schlafbezogener Atmungsstörungen als auch einem Basiswissen im Bereich der Schlafmedizin auszustatten, um diesen komplexen Patienten gerecht zu werden.

\section{Ziele}

Zweck des Curriculums ist die Beschreibung eines Qualifizierungsprozesses für spezialisierte Kompetenzen, dessen Ziel die Erlangung der Zusatzqualifikation Kardiovaskuläre Schlafmedizin ist. Damit soll die Qualifizierung in diesem spezialisierten Bereich verbessert werden, sodass sie über das Maß der allgemeinen Facharztweiterbildung in der Kardiologie hinausgeht.

In der curricularen Zusatzqualifizierung sollen in 3 Stufen folgende Kenntnisse und Fertigkeiten erlangt werden:

\section{Stufe 1}

Vermittlung der für Herz-Kreislauf-Erkrankungen relevanten Aspekte der Schlafmedizin [15]. Dies umfasst die Grundlagen in Ätiologie, Pathophysiologie, Pathogenese, Klinik, Diagnose und Differenzialdiagnose, Therapie und Differenzialtherapie sowie Prognose der für Herz-Kreislauf-Erkrankungen relevanten Aspekte der Atmungsund Schlafstörungen, v. a. der zentralen und obstruktiven Schlafapnoe, deren Diagnostik und Therapie, und Erkennen von Hinweisen und Prätestwahrscheinlichkeiten für schlafbezogene Atmungsstörungen aus üblichen kardiovaskulären Diagnostikverfahren.

\section{Stufe 2}

Erfahrung mit klinischen und vereinfachten apparativen diagnostischen Verfahren zur Stellung der Diagnose schlafbezogene Atmungsstörung, Differenzierung der respiratorischen Ereignisse in zentrale, obstruktive und gemischtförmige Anteile sowie ggf. Verfolgung weiterer Differenzialdiagnosen und einfache Therapiekontrollen. Dies beinhaltet die selbstständige Durchführung der Diagnostik von schlafbezogenen Atmungsstörungen mittels kardiorespiratorischer Polygraphie bei Patienten mit kardiovaskulären Erkrankungen unter Berücksichtigung differenzialdiagnostischer Aspekte sowie die Interpretation von Therapiegerätespeichern. Zudem werden fundierte Kenntnisse zu den Wechselwirkungen von schlafbezogenen Atmungsstörungen und kardiovaskulären Erkrankungen erwartet. 


\section{Stufe 3}

Selbstständige Durchführung der Diagnostik von schlafbezogenen Atmungsstörungen und deren Differenzialdiagnostik bei Patienten mit kardiovaskulären Erkrankungen sowie selbstständige Auswahl der richtigen Therapie, Durchführung der Therapieeinleitung und Therapieüberprüfung von schlafbezogenen Atmungsstörungen. Dies setzt fundierte Kenntnisse in der Durchführung und Interpretation der kardiorespiratorischen Polygraphie und Polysomnographie, aber auch detaillierte Kenntnisse und Erfahrungen mit allen zur Verfügung stehenden Therapieoptionen voraus.

Das Curriculum muss in einem definierten und von der DGK zertifizierten Prozess durchgeführt werden, welcher dem Qualitätsstandard der Zusatzqualifikation und der Qualifizierungsstätten zur Sicherung adäquater Qualifizierungsbedingungen Rechnung trägt.

\section{Syllabus}

\section{3. a) Lerninhalte}

Folgende Inhalte müssen während der 3-stufigen Zusatzqualifizierung in der kardiovaskulären Schlafmedizin vermittelt werden:

\section{Stufe 1}

Die Qualifizierungsinhalte der Stufe 1 entsprechen den Qualifizierungsinhalten des Curriculums Kardiologie [15]. Voraussetzung ist die Bereitschaft zur Integration der für Herzpatienten symptom- und prognoserelevanten Komponenten der Schlafmedizin in das kardiologische Therapiekonzept [15].

Im Einzelnen werden folgende theoretische Kenntnisse vermittelt:

- Definition, Subtypen und Schweregradeinteilung schlafbezogener Atmungsstörungen bei kardiovaskulären Erkrankungen,

- Pathophysiologie schlafbezogener Atmungsstörungen und Auswirkungen auf das kardiovaskuläre System,

- Indikationsstellung, Durchführung und Interpretation von vereinfachten applizierbaren (z. B. 2-Kanal-Systeme) und implantierbaren Diagnostiksys- temen (z. B. Schrittmachersysteme) zur Erfassung der Verdachtsdiagnose schlafbezogene Atmungsstörung,

- geeignete und ungeeignete Therapieverfahren für verschiedene Formen schlafbezogener Atmungsstörungen bei verschiedenen kardiologischen Erkrankungen.

Weiterhin werden folgende praktische Fähigkeiten vermittelt:

- spezielle Anamnese hinsichtlich der Hinweise auf eine schlafbezogene Atmungsstörung unter Berücksichtigung einer möglichen primären kardialen Symptomatik [15],

- Erfahrung mit vereinfachten apparativen Diagnostikverfahren sowie der kardiorespiratorischen Polygraphie (z. B. 2-Kanal-Systeme) zur Stellung der Verdachtsdiagnose Schlafapnoe und Verfolgung der weiterführenden Diagnostik einschließlich der Therapie (Kompetenzstufe 2 gemäß Curriculum Kardiologie) [15],

- Erkennen von Hinweisen und Prätestwahrscheinlichkeiten für schlafbezogene Atmungsstörungen aus üblichen kardiovaskulären Diagnostiken (Langzeitblutdruckmonitoring, Langzeit-EKG, Schrittmacher-/ICD-/CRTKontrollen, Spiroergometrie etc.).

\section{Stufe 2}

Kenntnisse zusätzlich zu Stufe 1:

- Anatomie der oberen Atemwege, Physiologie der Atmung, chronobiologische Grundlagen

- Pathophysiologie schlafbezogener Atmungsstörungen

- Akute pathophysiologische Konsequenzen schlafbezogener Atmungsstörungen auf das kardiovaskuläre System

- Chronische Effekte schlafbezogener Atmungsstörungen auf das kardiovaskuläre System und vice versa

- Patientenauswahl, Indikationen und Limitationen der Diagnostik schlafbezogener Atmungsstörungen

- Differenzialindikationen und Wertigkeit verfügbarer, diagnostischer Verfahren

- Verfügbare Therapieverfahren mit Indikationen und Kontraindikationen bei kardiologischen Krankheitsbildern
- Grundkenntnisse im Bereich der Polysomnographie

Fähigkeiten zusätzlich zu Stufe 1:

- Indikationsstellung, selbstständige Durchführung und Interpretation physiologischer und pathologischer Befunde in verschiedenen diagnostischen Verfahren: 1) vereinfachte applizierbare (z. B. 2-Kanal-Systeme) und implantierbare Diagnostiksysteme (z. B. Schrittmachersysteme) zur Erfassung der Verdachtsdiagnose schlafbezogene Atmungsstörung, 2) kardiorespiratorische Polygraphie zur Diagnose schlafbezogene Atmungsstörung, 3) Interpretation von Therapiespeichern interner oder externer Therapiegeräte

- Indikationsstellung für eine weiterführende Diagnostik in einer von der DGK anerkannten Stätte der Zusatzqualifikation (s. Abschn. 6) mit der Möglichkeit der kardiorespiratorischen Polysomnographie und atmungsspezifischen Differenzialtherapie

- Indikationsstellung einer Therapie von schlafbezogenen Atmungsstörungen

- Anpassen von Nasen-/Mund-NasenMasken zur Applikation verschiedener Formen der Überdruckbeatmungstherapie (CPAP, BiPAP, ASV, NIV) bei kardiovaskulär erkrankten Patienten

- Einleitung und Überprüfung dieser Therapieverfahren bei verschiedenen kardiologischen Erkrankungen

\section{Stufe 3}

Kenntnisse zusätzlich zu Stufe 2:

- Spezielle technische und pathophysiologische Grundlagen therapeutischer Verfahren

- Medikamentöse Therapiemaßnahmen

- Implantierbare Devices (kardiale Resynchronisationstherapie, transvenöse Phrenicusstimulation, Hypoglossusstimulation etc.)

- Operative Therapieverfahren

- Protrusionsschienen

- Fundierte Kenntnisse verschiedener nichtinvasiver Beatmungsverfahren (CPAP, BiPAP, ASV, NIV)

- Weiterführende Diagnoseverfahren (kardiorespiratorische Polysomnographie) 
- Technische Grundlagen

- Elektroenzephalographie

- Kardiorespiratorische Parameter, Sensorik, Aufzeichnung, Auswertung

- Begleitdiagnostik (EKG, Blutdruckmessung etc.)

- Management von Therapieeinleitung, Therapieoptimierung und Therapiekontrollen

- Psychosoziale Aspekte schlafbezogener Atmungsstörungen und deren Therapie

- Rechtliche Aspekte schlafbezogener Atmungsstörungen

Fähigkeiten zusätzlich zu Stufe 2:

- Selbstständige Durchführung und Interpretation von Polysomnographien inklusive Schlafstadienanalyse

- Konfiguration und praktische Handhabung üblicher Therapiedevices

- Selbstständige Einleitung und Überprüfung verschiedener Therapieverfahren bei kardiologischen Patienten mit schlafbezogenen Atmungsstörungen mittels kardiorespiratorischer Polygraphie und/oder Polysomnographie

- Selbstständiges Management von Komplikationen und Nebenwirkungen verschiedener Therapieverfahren

- Indikationsstellung für eine weiterführende Diagnostik in einem schlafmedizinischen Zentrum (z. B. Differenzialdiagnostik Insomnie, Narkolepsie und REM-assoziierte Verhaltensstörung)

- Selbstständiges Auslesen und Interpretation von Befunden aus implantierbaren Devices (Schrittmacher/ICD/CRT)

- Selbstständige Erhebung und Interpretation von Befunden der kardiopulmonalen Funktionsdiagnostik (Langzeit-EKG, Langzeitblutdruckmonitoring, Echokardiographie, Lungenfunktionsanalyse, Spiroergometrie, Blutgasanalyse etc.) im Hinblick auf das Vorhandensein von schlafbezogenen Atmungsstörungen bzw. deren Therapieeffektivität

- Selbstständige Erhebung und Interpretation von invasiven und nichtinvasiven, kontinuierlichen Hämodynamikmessungen bei kardiologischen Patienten mit schlafbezogenen At- mungsstörungen mit und ohne Therapie

\section{3. b) Lernziele}

\section{Stufe 1}

Erwerb von Grundkenntnissen in den Bereichen:

- Definition, Subtypen und Schweregradeinteilung schlafbezogener Atmungsstörungen bei kardiovaskulären Erkrankungen

- Pathophysiologie schlafbezogener Atmungsstörungen und Auswirkungen auf das kardiovaskuläre System

- Indikationsstellung, Durchführung und Interpretation von klinischen und vereinfachten apparativen diagnostischen Verfahren zur Stellung der Verdachtsdiagnose schlafbezogene Atmungsstörung

- Allgemeine Kenntnisse zu Therapieverfahren für verschiedene Formen schlafbezogener Atmungsstörungen bei kardiologischen Patienten

- Erkennen von Hinweisen und Prätestwahrscheinlichkeiten für schlafbezogene Atmungsstörungen aus üblichen kardiovaskulären Diagnostiken (Langzeitblutdruckmonitoring, Langzeit-EKG, Echokardiographie, Schrittmacher-/ICD-/CRT-Kontrollen, Spiroergometrie etc.)

\section{Stufe 2}

- Indikationsstellung zur Durchführung einer kardiorespiratorischen Polygraphie oder kardiorespiratorischen Polysomnographie bei Verdacht auf Vorliegen schlafbezogener Atmungsstörungen

- Selbstständiges Anlegen und Durchführung von vereinfachten diagnostischen Verfahren (z. B. 2-Kanal-Systeme, Langzeit-EKG-Systeme)

- Selbstständiges Anlegen und Durchführung einer kardiorespiratorischen Polygraphie

- Selbstständige Auswertung und Interpretation verschiedener vereinfachter diagnostischer Verfahren zur Stellung der Verdachtsdiagnose schlafbezogene Atmungsstörung

- Selbstständige Auswertung und Interpretation einer Diagnostik mittels kardiorespiratorischer Polygraphie
- Indikationsstellung für eine weiterführende Diagnostik in einem schlafmedizinischen Zentrum (z. B. Differenzialdiagnostik Insomnie, Narkolepsie und REM-schlafbezogene Verhaltensstörung)

- Befundvermittlung an den Patienten unter Berücksichtigung psychosozialer und rechtlicher Aspekte

- Selbstständiges Abfragen von implantierbaren Devices zur Erkennung schlafbezogener Atmungsstörungen

- Selbstständiges Auslesen und Interpretation von Speichern implantierter bzw. nicht implantierter Therapiesysteme

Stufe 3

- Selbstständige Auswertung und Interpretation einer Diagnostik und Therapieeinleitung mittels kardiorespiratorischer Polygraphie und/oder Polysomnographie

- Selbstständige Indikationsstellung und Differenzialindikationen für verschiedene Therapieverfahren bei schlafbezogenen Atmungsstörungen einschließlich komplexer Fälle

- Sicheres Beherrschen von Konfigurationen und praktischer Handhabung üblicher Therapieverfahren (z. B. CPAP, BiPAP, ASV, NIV)

- Selbstständige Auswertung und Interpretation einer Therapieeinleitung von schlafbezogenen Atmungsstörungen bei Patienten mit kardiovaskulären Erkrankungen mittels kardiorespiratorischer Polygraphie und/oder Polysomnographie

- Management von Therapieeinleitung, Therapieoptimierung und Therapiekontrollen inklusive Nebenwirkungen und Komplikationen

- Selbstständige Auswertung und Interpretation einer Therapieüberprüfung von schlafbezogenen Atmungsstörungen bei Patienten mit kardiovaskulären Erkrankungen mittels kardiorespiratorischer Polygraphie und/oder Polysomnographie

- Indikationsstellung für eine weiterführende Differenzialdiagnostik in einem schlafmedizinischen Zentrum

- Selbstständige Erhebung und Interpretation von invasiven und nichtinvasiven Hämodynamikbefunden bei 
kardiologischen Patienten mit schlafbezogenen Atmungsstörungen mit und ohne Therapie

\section{Durchführung der Qualifizierung}

Die Qualifizierung erfolgt gemäß den oben aufgeführten Lernzielen in 3 Stufen. Jede Qualifizierungsstufe muss an einer von der DGK für die Zusatzqualifikation Kardiovaskuläre Schlafmedizin anerkannten Stätte durchgeführt werden (s. Abschn. 6). Eine Absolvierung der Qualifizierung durch Hospitation an einer von der DGK zertifizierten Stätte der Zusatzqualifikation ist möglich.

Die Dauer der Qualifizierung und die von den Programmkandidaten zu erfüllenden Kriterien und einzureichenden Dokumente und Nachweise für die drei Qualifizierungsstufen sind in - Tab.2 unter Abschn. 12 zusammengefasst. Programmkandidaten aus dem Ausland müssen die Gleichwertigkeit ihrer kardiologischen Facharztweiterbildung durch eine Urkunde der jeweiligen Landesärztekammer nachweisen.

\section{Stufe 1}

In der Stufe 1 erfolgt eine Einführung in schlafmedizinische Grundbegriffe und pathophysiologische Zusammenhänge sowie die Einführung in eine schlafmedizinische Anamnese und körperliche Untersuchung bei kardiovaskulär erkrankten Patienten:

- Pathophysiologie schlafbezogener Atmungsstörungen,

- diagnostische Möglichkeiten und wichtige Differenzialdiagnosen (Insomnie, psychiatrische Schlafstörungen u.a.),

- Therapiemöglichkeiten der Schlafapnoe.

Dies erfolgt durch die Teilnahme am Grundkurs Kardiovaskuläre Schlafmedizin (4 CME-Punkten entsprechend, z.B. im Rahmen der DGK-Akademie) während der Programmteilnahme sowie durch die aktive klinische Tätigkeit.

Kursinhalte

- Definition, Subtypen und Schweregradeinteilung schlafbezogener At- mungsstörungen bei kardiovaskulären Erkrankungen

- Pathophysiologie schlafbezogener Atmungsstörungen und Auswirkungen auf das kardiovaskuläre System

- Indikationsstellung, Durchführung und Interpretation von vereinfachten applizierbaren (z. B. 2-Kanal-Systeme) und implantierbaren Diagnostiksystemen (z. B. Schrittmachersysteme) zur Erfassung der Verdachtsdiagnose schlafbezogene Atmungsstörung

- Geeignete und ungeeignete Therapieverfahren für verschiedene Formen schlafbezogener Atmungsstörungen bei verschiedenen kardiologischen Erkrankungen

Die Fähigkeiten werden an der Qualifizierungsstätte vermittelt und theoretisch anhand von Fallbeispielen im Grundkurs kardiovaskuläre Schlafmedizin unterstützt:

- spezielle Anamnese hinsichtlich der Hinweise auf eine schlafbezogene Atmungsstörung unter Berücksichtigung einer möglichen primären kardialen Symptomatik [15],

- Erfahrung mit vereinfachten apparativen Diagnostikverfahren sowie der kardiorespiratorischen Polygraphie (z. B. 2-Kanal-Systeme) zur Stellung der Verdachtsdiagnose Schlafapnoe und Verfolgung der weiterführenden Diagnostik einschließlich der Therapie (Kompetenzstufe 2 gemäß Curriculum Kardiologie) [15],

- Erkennen von Hinweisen und Prätestwahrscheinlichkeiten für schlafbezogene Atmungsstörungen aus üblichen kardiovaskulären Diagnostiken (Langzeitblutdruckmonitoring, LangzeitEKG, Schrittmacher-/ICD-/CRT-Kontrollen, Spiroergometrie etc.).

Im Rahmen der klinischen Tätigkeit müssen mindestens 50 Patienten mit kardiovaskulären Erkrankungen und Verdacht auf schlafbezogene Atmungsstörungen (SBAS) mitbetreut und deren Behandlung mittels eines von der DGK zur Verfügung gestellten Logbuchs nachgewiesen werden.

\section{Stufe 2}

Die Vermittlung der über die Stufe 1 hinausgehenden theoretischen Kenntnisse erfolgt im Rahmen von fachspezifischen Vorträgen und der Teilnahme am Aufbaukurs Kardiovaskuläre Schlafmedizin über insgesamt 30 CME-Punkte während der Programmteilnahme (an 2 Wochenenden analog BUB-Kurs).

\section{Kursinhalte}

- Anatomie der oberen Atemwege, Physiologie der Atmung, chronobiologische Grundlagen

- Pathophysiologie schlafbezogener Atmungsstörungen

- Akute pathophysiologische Konsequenzen schlafbezogener Atmungsstörungen auf das kardiovaskuläre System

- Chronische Effekte schlafbezogener Atmungsstörungen auf das kardiovaskuläre System und vice versa

- Patientenauswahl, Indikationen und Limitationen der Diagnostik schlafbezogener Atmungsstörungen

- Differenzialindikationen und Wertigkeit verfügbarer, diagnostischer Verfahren

- verfügbare Therapieverfahren mit Indikationen und Kontraindikationen bei kardiologischen Krankheitsbildern

- Grundkenntnisse im Bereich der Polysomnographie

Vermittelte Fähigkeiten im Rahmen von Hands-on-Workshops als Teil des Aufbaukurs Kardiovaskuläre Schlafmedizin

- Indikationsstellung, selbstständige Durchführung und Interpretation physiologischer und pathologischer Befunde in verschiedenen diagnostischen Verfahren:

1. Vereinfachte applizierbare (z. B. 2-Kanal-Systeme) und implantierbare Diagnostiksysteme (z. B. Schrittmachersysteme) zur Erfassung der Verdachtsdiagnose schlafbezogene Atmungsstörung

2. Kardiorespiratorische Polygraphie zur Diagnose schlafbezogene Atmungsstörung 
3. Interpretation von Therapiespeichern interner oder externer Therapiegeräte

- Indikationsstellung für eine weiterführende Diagnostik in einer von der DGK anerkannten Stätte der Zusatzqualifikation (s. Abschn. 6) mit der Möglichkeit der kardiorespiratorischen Polysomnographie und atmungsspezifischen Differenzialtherapie

- Indikationsstellung einer Therapie von schlafbezogenen Atmungsstörungen

- Anpassen von Nasen-/Mund-NasenMasken zur Applikation verschiedener Formen der Überdruckbeatmungstherapie (CPAP, BiPAP, ASV, NIV) bei kardiovaskulär erkrankten Patienten

- Einleitung und Überprüfung dieser Therapieverfahren bei verschiedenen kardiologischen Erkrankungen

Die Stufe 2 beinhaltet zusätzlich obligat eine Qualifizierungszeit von mindestens 2 Wochen in Vollzeit an einer von der DGK anerkannten Stätte (s. Abschn. 6).

Dabei müssen mindestens

- 30 schlafmedizinische Fälle einschließlich Anamnese, Diagnostik und Therapieeinleitung dokumentiert werden;

- 30 Polygraphien selbstständig und

- 30 Polysomnographien unter Anleitung/Supervision befundet werden, davon mindestens

- 10 Polygraphien/Polysomnographien mit obstruktiver Schlafapnoe und

- 10 Polygraphien/Polysomnographien mit zentraler Schlafapnoe;

- 10 Therapiekontrollen (Geräteauslesung, Polygraphie oder Polysomnographie) bei Patienten mit einer schlafbezogenen Atmungsstörung erfolgen.

Die Prozeduren müssen in einem von der DGK zur Verfügung gestellten Logbuch dokumentiert und durch den (stellv.) Leiter der Zusatzqualifikation bestätigt werden.

\section{Stufe 3}

In der Stufe 3 muss der Programmkandidat zusätzlich zu den in Stufe 2 erworbenen theoretischen Kenntnissen 6 Monate in Vollzeit an einer Stätte der Zusatzqualifikation (s. Abschn. 6) tätig sein, davon müs- sen mindestens 3 Monate Vollzeittätigkeit im Schlaflabor durchgeführt werden.

In dieser Zeit müssen mindestens 150 schlafmedizinische Untersuchungen ( $\geq 100$ diagnostische Polysomnographien, $\geq 50$ Polysomnographien zur Einleitung einer Positivdrucktherapie) durchgeführt werden, wobei diese Untersuchungen anhand eines von der DGK zur Verfügung gestellten Logbuchs nachzuweisen sind. Von diesen 150 Untersuchungen müssen alle selbstständig befundet worden sein einschließlich Anamnese, Untersuchung, Befundung der schlafmedizinischen Untersuchung, Therapieeinleitung und Nachsorgeempfehlung.

\section{Anforderungen an den Programmkandidaten}

Voraussetzung vor Beginn der Qualifizierungszeit für die Stufen 1, 2 und 3 ist die Approbation als Arzt und der Beginn der Weiterbildung im Bereich Innere Medizin bzw. Innere Medizin und Kardiologie.

\section{Stufe 1}

Die Qualifizierungszeit in der kardiovaskulären Schlafmedizin ist Teil der Weiterbildung des Curriculums Kardiologie [14, 15]. Voraussetzung vor Beginn der Qualifizierungszeit für Stufe 1 ist die Approbation als Arzt und der Beginn der Weiterbildung im Bereich Innere Medizin bzw. Innere Medizin und Kardiologie. Die Antragstellung zur persönlichen Anerkennung für Stufe 1 ist jedoch erst nach Abschluss der kardiologischen Facharztweiterbildung mit den erforderlichen Dokumenten und Qualifizierungsnachweisen, insbesondere der Facharzturkunde Kardiologie, möglich (s. - Tab. 2, Abschn. 12).

Die Maximaldauer der Programmteilnahme für Stufe 1 beträgt 24 Monate.

\section{Stufe 2 und 3}

Die Qualifizierung muss Kenntnisse in den kardiologischen Krankheitsbildern, der Pathophysiologie und der kardiologischen Schlafmedizin (Anamnese, Fragebögen, Polygraphie) beinhalten. Die Qualifizierung vermittelt Erfahrungen in der Betreuung von Patienten mit internistischen und komplexen kardiologischen Erkrankungen sowie die diagnostischen und therapeutischen Maßnahmen bei kardiovaskulären Erkrankungen. Die Sachkundenachweise für Herzschrittmachertherapie, ICD-Therapie und CRT-Therapie sollen vorliegen.

Die Antragstellung zur persönlichen Anerkennung für Stufe 2 und 3 erfolgt mit den erforderlichen Dokumenten und Qualifizierungsnachweisen, insbesondere der Facharzturkunde Kardiologie (s. Abschn. 12). Eine Absolvierung der Qualifizierung in Teilzeit (mindestens 50\% einer Vollzeitstelle) ist möglich. In diesem Fall verlängert sich der Qualifizierungszeitraum um die entsprechende Zeit. Die Qualifizierung für Stufe 2 kann in Blöcken mit einer Minimalzeit von 2-mal 1 Woche in Vollzeit (bei Teilzeit entsprechend länger) absolviert werden. Für Stufe 3 ist eine Absolvierung der Qualifizierung in Blöcken von jeweils 3 Monaten in Vollzeit (bei Teilzeit ebenfalls entsprechend länger) möglich.

Die Maximaldauer der Programmteilnahme für Stufe 2 beträgt 3 Monate und 24 Monate für Stufe 3.

Die einzelnen Qualifizierungsstufen bauen thematisch aufeinander auf. Die Antragstellung erfolgt für die Stufe, die am Ende der Qualifizierung erreicht werden soll, unter der Bedingung, dass alle Voraussetzungen für die vorangehenden Stufen erfüllt werden. Eine Qualifizierung für Stufe 2 und 3 ist demnach erst nach Absolvierung der jeweils vorherigen Stufe möglich.

Die persönlichen Anerkennungen (Stufen 1-3) werden befristet erteilt; anschließend ist eine Rezertifizierung notwendig.

\section{Anforderungen an die Stätte der Zusatzqualifikation}

Als Stätte der Zusatzqualifikation sind sowohl stationäre als auch ambulante Einrichtungen geeignet, die sowohl über die Möglichkeit der kardiologischen Diagnostik (einschließlich der Abfrage von Schrittmacher-, CRT- und ICD-Systemen) und Therapie als auch der Polygraphie, Polysomnographie und Therapieeinleitung bzw. Therapiekontrolle schlafbezogener Atmungsstörungen verfügen. Die Qualifizierung ist auch in interdisziplinären Einrichtungen möglich, sofern die Einrichtung DGK-zertifiziert ist. Dabei ist es 
unerheblich, ob diese Einrichtungen ganz oder teilweise ambulant arbeiten. Wichtig sind der kardiologische Schwerpunkt, die Qualifikation des (stellv.) Leiters der Zusatzqualifikation entsprechend Abschn. 7 und die im Folgenden beschriebene Möglichkeit der Polygraphie, Polysomnographie, Therapieeinleitung und Therapienachsorge sowie die Einhaltung von Mindestzahlen. Für die Vorbereitung der Patienten und Durchführung der Untersuchungen muss entsprechend spezialisiertes, in schlafmedizinischer Untersuchung und Maskenanpassung geschultes Personal (mindestens 1 MTA/MFA oder eine Fachkraft mit vergleichbarer Ausbildung) arbeitstäglich vorhanden sein.

Die Stätte der Zusatzqualifikation muss das übliche Spektrum an kardiovaskulären Erkrankungen mit einem Minimum von 250 Polygraphien und/oder Polysomnographien pro Jahr abbilden. Weiterhin sind 100 Therapieeinleitungen und mindestens 100 Therapiekontrollen pro Jahr erforderlich (s. - Tab. 4, Abschn. 12). Für die Durchführung der Untersuchungen muss ein entsprechendes Team vorhanden sein, bestehend aus einem persönlich für Stufe 3 anerkannten (stellv.) Leiter der Zusatzqualifikation sowie MTAs, Krankenpflegern oder medizinischen Fachangestellten, die überwiegend in der schlafmedizinischen Diagnostik und Therapie arbeiten und entsprechend dem Medizinproduktgesetz in alle erforderlichen Geräte (Polygraphie, Polysomnographie, Positivdruckgeräte) eingewiesen sind (s. - Tab.4, Abschn. 12).

Die Stätte muss über schlafmedizinische Vorrichtungen im Sinne von Polysomnographiemessplätzen (>1 Einzelzimmer) für Patienten verfügen (s. - Tab. 4, Abschn. 12). Auch Messplätze außerhalb des Krankenhauses werden anerkannt.

Fallkonferenzen mit dem (stellv.) Leiter der Zusatzqualifikation müssen wöchentlich gewährleistet sein. Darüber hinaus muss die Stätte regelmäßig (mindestens 2-mal/Jahr) schlafmedizinische Fortbildungen für die Mitarbeiter durchführen.

Die Anerkennung erfolgt nach Einreichung entsprechender Unterlagen und Leistungszahlen und ist nur in Zusammenhang mit der Anerkennung eines Leiters und eines stellv. Leiters möglich (s. Abschn. 7).
Die mit dem Stätteantrag einzureichenden Dokumente und Nachweise sind in - Tab. 4 unter Abschn. 12 zusammengefasst.

Die Anerkennung als Stätte der Zusatzqualifikation wird befristet erteilt; anschließend ist eine Rezertifizierung notwendig.

\section{Anforderungen an den (stellv.) Leiter der Zusatzqualifikation}

Um eine Qualifizierung gemäß dem Curriculum Kardiovaskuläre Schlafmedizin sicherzustellen, sind besondere Voraussetzungen an den Leiter und den zusätzlich erforderlichen stellv. Leiter der Zusatzqualifikation sowie an die Stätte (s. Abschn. 6) zu stellen.

Die vom (stellv.) Leiter der Zusatzqualifikation zu erfüllenden Kriterien und einzureichenden Dokumente und Nachweise sind in DTab. 5 unter Abschn. 12 zusammengefasst.

Der (stellv.) Leiter der Zusatzqualifikation muss Facharzt für Innere Medizin mit Schwerpunkt Kardiologie bzw. Innere Medizin und Kardiologie sein und detaillierte Kenntnisse im Bereich kardiovaskuläre Schlafmedizin besitzen. Darüber hinaus muss der (stellv.) Leiter der Zusatzqualifikation über die persönliche Anerkennung der Zusatzqualifikation Stufe 3 verfügen und in Vollzeit an der Stätte tätig sein.

Optional gilt folgende Regelung: Der Leiter des kooperierenden Schlaflabors und Leiter der Zusatzqualifikation muss entweder "Facharzt für Allgemeinmedizin", "Facharzt für Hals-Nasen-OhrenHeilkunde", "Facharzt für Innere Medizin", „Facharzt für Innere Medizin und Pneumologie" (auch Anerkennung als „Facharzt für Innere Medizin“ in Verbindung mit der Schwerpunktbezeichnung „Pneumologie“ nach bisherigem Recht), „Facharzt für Neurologie" (auch „Facharzt für Nervenheilkunde" nach bisherigem Recht) oder „Facharzt für Psychiatrie und Psychotherapie" sein und detaillierte Kenntnisse im Bereich kardiovaskuläre Schlafmedizin besitzen. Darüber hinaus muss der Leiter der Zusatzqualifikation in Vollzeit an der Stätte tätig sein. In diesem Fall muss der stellv. Leiter der Zusatzqualifikation Facharzt für Innere Medizin mit Schwerpunkt Kardiologie bzw. Innere Medizin und Kardiologie sein und detaillierte Kenntnisse im Bereich kardiovaskuläre Schlafmedizin besitzen. Darüber hinaus muss der stellv. Leiter der Zusatzqualifikation über die persönliche Anerkennung der Zusatzqualifikation Stufe 3 verfügen und in Vollzeit an der Stätte tätig sein (• Tab. 5).

Mindestens der Leiter oder der (stellv.) Leiter der Zusatzqualifikation soll den Sachkundenachweis für Herzschrittmachertherapie, ICD-Therapie und CRT-Therapie haben.

Der (stellv.) Leiter der Zusatzqualifikation muss in den letzten 5 Jahren vor Antragstellung mindestens 50 vereinfachte apparative diagnostische Verfahren zur Stellung der Verdachtsdiagnose schlafbezogene Atmungsstörung pro Jahr (z. B. 1- bis 2-KanalAufzeichnungen und/oder Aufzeichnungen aus implantierbaren elektrischen Devices) sowie weitere 50 kardiorespiratorische Polygraphien pro Jahr initiiert und interpretiert haben. Zudem muss er in den letzten 5 Jahren mindestens 50 Therapieeinleitungen pro Jahr sowie 50 Therapiekontrollen pro Jahr verantwortet haben.

Der (stellv.) Leiter der Zusatzqualifikation ist angehalten, die DGK umgehend darüber zu informieren, wenn er die Stätte der Zusatzqualifikation verlässt. In jedem Fall aber ist die Stätte verpflichtet, den Weggang eines (stellv.) Leiters möglichst vor Beendigung des Beschäftigungsverhältnisses, spätestens jedoch 4 Wochen danach, schriftlich bei der DGK zu melden. Der Antrag für einen neuen (stellv.) Leiter ist spätestens innerhalb von 6 Monaten nach Ausscheiden des bisherigen (stellv.) Leiters schriftlich einzureichen. Andernfalls wird der Stätte die Zertifizierung aberkannt.

\section{Evaluierung}

Die Evaluierung der Programmkandidaten setzt sich aus 3 Komponenten zusammen:

\section{Dokumentation der Qualifizierung}

a) Beleg der kontinuierlichen Qualifizierung in der Subdisziplin durch Nachweis der Teilnahme an akkreditierten Kongressen, Workshops, Symposien, Seminaren, Hospitationen oder Trainingskursen der Fachgesellschaften oder ihrer Mitglieder. 
Tab. 1 Persönliche Anerkennung der Zusatzqualifikation Kardiovaskuläre Schlafmedizin im Rahmen der Übergangsregelung - Übersicht der Voraussetzungen und Kriterien

\begin{tabular}{|c|c|c|c|}
\hline $\begin{array}{l}\text { Anzu- } \\
\text { erken- } \\
\text { nende } \\
\text { Stufe }\end{array}$ & Voraussetzungen & Mindestzahlen & Einzureichende Unterlagen \\
\hline 3 & $\begin{array}{l}\text { - Facharztanerken- } \\
\text { nung Innere Me- } \\
\text { dizin und Kar- } \\
\text { diologie bzw. } \\
\text { Innere Medizin } \\
\text { mit Schwerpunkt } \\
\text { Kardiologie } \\
\text { - Teilnahme } \\
\text { BUB-Kurs oder } \\
\text { gültige KV-Aner- } \\
\text { kennung zur Ab- } \\
\text { rechnung schlaf- } \\
\text { medizinischer } \\
\text { Leistungen }\end{array}$ & $\begin{array}{l}\text { Durchführung und } \\
\text { selbstständige Be- } \\
\text { fundung von } 150 \\
\text { schlafmedizinischen } \\
\text { Untersuchungen, da- } \\
\text { von: } \\
\text { - mindestens } 100 \\
\text { diagnostische Poly- } \\
\text { somnographien } \\
\text { - mindestens } 50 \text { Po- } \\
\text { lysomnographien } \\
\text { zur Einleitung einer } \\
\text { Positivdrucktherapie }\end{array}$ & $\begin{array}{l}\text { - Aktueller Lebenslauf } \\
\text { - Facharzturkunde Innere Medi- } \\
\text { zin und Kardiologie bzw. Innere } \\
\text { Medizin mit Schwerpunkt Kar- } \\
\text { diologie } \\
\text { - Zeugnis des Leiters der Einrich- } \\
\text { tung über die überwiegende } \\
\text { Tätigkeit im Bereich Schlaf- und } \\
\text { Beatmungsmedizin sowie die } \\
\text { geforderten Mindestzahlen } \\
\text { - Teilnahmebescheinigung } \\
\text { BUB-Kurs oder gültige KV-An- } \\
\text { erkennung }\end{array}$ \\
\hline
\end{tabular}

b) Dokumentation der durchgeführten Untersuchungen, Therapieeinleitungen und Therapienachsorgen in einem von der DGK zur Verfügung gestellten Logbuch. Sämtliche Prozeduren und die verantwortliche Stellung (, gesehen“, „in Assistenz", „unter Anleitung eines Stufe-3-Mentors" oder "selbstständig durchgeführt") müssen dabei dokumentiert werden. Die Korrektheit des Logbuchs wird schriftlich durch den Leiter oder den stellv. Leiter der Zusatzqualifikation bestätigt.

c) Bericht des Leiters der Zusatzqualifikation

Der Bericht des Leiters der Zusatzqualifikation zum Erwerb der Stufe 1 bescheinigt die Einführung in das Thema sowie Grundkenntnisse in Pathophysiologie, klinischer Bedeutung, Diagnose und Differenzialdiagnose sowie allgemeinen Therapieverfahren schlafbezogener Atmungsstörungen. Mittels Logbucheintrag wird die Mitbetreuung von mindestens 50 Patienten mit der Verdachtsdiagnose einer schlafbezogenen Atmungsstörung belegt.

Berichte des Leiters der Zusatzqualifikation zum Erwerb der Stufe 2 und 3 sind detaillierter anzufertigen. Diese müssen Details zu den Aktivitäten, der Kompetenz und der erreichten Selbstständigkeit des Programmkandidaten beinhalten. Diese Berichte enthalten also neben Informationen zu den Kenntnissen und Erfahrungen auch eine Beschreibung der Fortschritte in praktischen Tätigkeiten und theoretischem Wissen. Es muss erkennbar sein, dass der Programmkandidat als unabhängiger Untersucher diagnostische Verfahren indiziert, selbstständig durchgeführt und ausgewertet hat. Therapeutische Konsequenzen sowie differenzialdiagnostische und -therapeutische Überlegungen müssen sich an den neuesten klinisch-wissenschaftlichen Erkenntnissen orientieren. Die Interaktion mit dem Team, dem Patienten und den Angehörigen muss beschrieben werden.

Zur Anerkennung sind die oben genannten Bescheinigungen der erfolgreichen Teilnahme an akkreditierten Fortbildungen, Kongressen, Symposien (jeweils mindestens 4 CME-Punkte für Stufe 1 und 30 CME-Punkte über den gesamten Qualifizierungszeitraum für Stufe 2 und 3) u.a. zusammen mit dem Logbuch und dem Bericht des Leiters der Zusatzqualifikation über den gesamten Qualifizierungszeitraum einzureichen (s. - Tab. 2 , Abschn. 12).

Anträge auf persönliche Anerkennung der Zusatzqualifikation werden durch die vom Vorstand der DGK benannten Gutachter der Zusatzqualifikation geprüft. Die Antragstellung erfolgt ausschließlich online. Einzureichen sind ein aktueller Lebenslauf, die Facharzturkunde Kardiologie, die Dokumentation der kontinuierlichen Qualifizierung in der Subdisziplin, das von der
DGK zur Verfügung gestellte Logbuch und die Beurteilung des Leiters der Zusatzqualifikation. Die persönliche Anerkennung der Zusatzqualifikation wird befristet erteilt. Danach muss für die Fortdauer der Anerkennung eine Rezertifizierung erfolgen.

Die Anerkennung als Stätte der Zusatzqualifikation und als (stellv.) Leiter der Zusatzqualifikation erfolgt durch die DGK in Zusammenarbeit mit den jeweiligen Gutachtern. Die Anerkennung der Stätte wird durch den zukünftigen Leiter der Zusatzqualifikation beantragt und nach Begutachtung der eingereichten Unterlagen durch die vom Vorstand der DGK benannten Gutachter bei Erfüllung der Voraussetzungen gewährt. Die Antragstellung erfolgt ausschließlich online. Dem Stätteantrag ist eine ausführliche Beschreibung der Stätte beizufügen, die Aufschluss über die personelle, räumliche und apparative Ausstattung sowie den Ablauf und die Struktur in der Stätte gibt. Weiterhin sind Angaben zu Konferenzen und internen sowie ggf. externen Fortbildungen beizufügen.

Räumliche, personelle und apparative Voraussetzungen an die Stätte der Zusatzqualifikation sind (modifiziert nach [13]):

- Eigene Räume, die als Einzelplatzräume zur Durchführung von Polysomnographien ausgestattet sind.

- Polysomnographiemessplätze müssen ausreichend abdunkelbar, schallabgeschirmt ( $<40-45 \mathrm{~dB})$, belüftungsfähig und temperierbar sein; mindestens 1 Zimmer muss behindertengerecht ausgestattet sein.

- Personelle Voraussetzungen sind ein Leiter und ein stellv. Leiter der Zusatzqualifikation sowie mindestens 1 MTA/MFA/Pfleger mit Erfahrung in der Polysomnographie/ Therapieeinleitung im Tag- sowie im Nachtdienst.

- Das Personal für die nächtlichen Ableitungen (hier Nachtwachen genannt) muss permanent während der nächtlichen Messung anwesend sein.

- Eine Nachtwache darf nicht mehr als 4 Patienten versorgen.

- Der ärztliche Dienst muss zu den Zeiten des Patientenbetriebs der Polygraphieund Polysomnographiemessplätze bei Notfällen zeitnah zur Verfügung stehen. 
Tab. 2 Persönliche Anerkennung der Zusatzqualifikation Kardiovaskuläre Schlafmedizin durch Programmteilnahme - Übersicht der Voraussetzungen und Kriterien für die 3 Stufen der Qualifizierung

\begin{tabular}{|c|c|c|c|c|}
\hline Stufe & Voraussetzungen & Mindestzahlen & Qualifizierungsinhalt & $\begin{array}{l}\text { Einzureichende Un- } \\
\text { terlagen }\end{array}$ \\
\hline 1 & $\begin{array}{l}\text { Approbation als Arzt und Beginn } \\
\text { der Weiterbildung zum Facharzt } \\
\text { Innere Medizin und Kardiologie } \\
\text { bzw. Innere Medizin mit Schwer- } \\
\text { punkt Kardiologie } \\
\text { Strukturierte Qualifizierung im } \\
\text { Rahmen von Vorträgen: } \\
\text { 4 CME-Punkte (z. B. } \\
\text { DGK-Akademie) }\end{array}$ & $\begin{array}{l}\text { Mitbetreuung von mindestens } 50 \text { Patienten mit } \\
\text { kardiovaskulären Erkrankungen und Verdacht auf } \\
\text { schlafbezogene Atmungsstörungen } \\
\text { Mentor: Facharzt für Innere Medizin und Kar- } \\
\text { diologie bzw. Innere Medizin mit Schwerpunkt } \\
\text { Kardiologie mit persönlicher Anerkennung der } \\
\text { Stufe } 3 \text { der Zusatzqualifikation } \\
\text { Optional gilt folgende Regelung: Leiter des ko- } \\
\text { operierenden Schlaflabors mit detaillierten } \\
\text { Kenntnissen in der kardiovaskulären Schlafme- } \\
\text { dizin (s. Abschn. 7) } \\
\text { Maximaldauer der Programmteilnahme: } \\
24 \text { Monate }\end{array}$ & $\begin{array}{l}\text { - Grundlagen: Pa- } \\
\text { thophysiologie } \\
\text { schlafbezogener } \\
\text { Atmungsstörungen } \\
\text { - Grundlagen: diag- } \\
\text { nostische Möglich- } \\
\text { keiten und wichtige } \\
\text { Differenzialdiagno- } \\
\text { sen } \\
\text { - Grundlagen: Thera- } \\
\text { piemöglichkeiten } \\
\text { der Schlafapnoe } \\
\text { - Allgemeinwissen } \\
\text { des Kardiologen zur } \\
\text { Anwendung in der } \\
\text { Praxis oder Klinik }\end{array}$ & $\begin{array}{l}\text { - Aktueller Lebenslauf } \\
\text { - Facharzturkunde } \\
\text { Innere Medizin und } \\
\text { Kardiologie bzw. } \\
\text { Innere Medizin } \\
\text { mit Schwerpunkt } \\
\text { Kardiologie } \\
\text { - Logbuch } \\
\text { - Detailliertes Zeugnis } \\
\text { des (stellv.) Leiters } \\
\text { der Zusatzqualifika- } \\
\text { tion mit Angabe des } \\
\text { Qualifizierungszeit- } \\
\text { raums, der durchge- } \\
\text { führten Prozeduren, } \\
\text { der Aktivitäten } \\
\text { und erworbenen } \\
\text { Kompetenz und } \\
\text { erreichten Selbst- } \\
\text { ständigkeit } \\
\text { - Nachweis der } \\
\text { CME-Punkte }\end{array}$ \\
\hline 2 & $\begin{array}{l}\text { Strukturierte Qualifizierung im } \\
\text { Rahmen von Vorträgen im Rah- } \\
\text { men der Weiterbildungsakademie: } \\
30 \text { CME-Punkte oder Analog } \\
\text { BUB-Kurs } \\
\text { 2-wöchige Tätigkeit in Vollzeit an } \\
\text { einer von der DGK nach Curricu- } \\
\text { lum Kardiovaskuläre Schlafmedi- } \\
\text { zin zertifizierten Stätte }\end{array}$ & $\begin{array}{l}\text { Logbuch-Nachweis: } \\
-\geq 30 \text { Fälle kardiovaskuläre Schlafmedizin ein- } \\
\text { schließlich Anamnese, Diagnostik und Therapie- } \\
\text { einleitung } \\
-\geq 30 \text { Polygraphien (selbstständig) } \\
\text { - } \geq 30 \text { Polysomnographien unter Anleitung, da- } \\
\text { von: a) } 10 \text { Polygraphien/Polysomnographien mit } \\
\text { obstruktiver Schlafapnoe und b) } 10 \text { Polygraphien/ } \\
\text { Polysomnographien mit zentraler Schlafapnoe } \\
\text { - } 10 \text { Therapiekontrollen bei Patienten mit einer } \\
\text { schlafbezogenen Atmungsstörung } \\
\text { Mentor: Facharzt für Innere Medizin und Kar- } \\
\text { diologie bzw. Innere Medizin mit Schwerpunkt } \\
\text { Kardiologie mit persönlicher Anerkennung der } \\
\text { Stufe } 3 \text { der Zusatzqualifikation } \\
\text { Optional gilt folgende Regelung: Leiter des ko- } \\
\text { operierenden Schlaflabors mit detaillierten } \\
\text { Kenntnissen in der kardiovaskulären Schlafme- } \\
\text { dizin (s. Abschn. 7) } \\
\text { Maximaldauer der Programmteilnahme: } \\
3 \text { Monate }\end{array}$ & $\begin{array}{l}\text { - Vertiefte Grundla- } \\
\text { gen: Pathophysio- } \\
\text { logie schlafbezo- } \\
\text { gener Atmungsstö- } \\
\text { rungen } \\
\text { - Fundierte Kenntnis- } \\
\text { se diagnostischer } \\
\text { Möglichkeiten und } \\
\text { wichtiger Differen- } \\
\text { zialdiagnosen } \\
\text { - Selbstständige Dia- } \\
\text { gnostik schlafbezo- } \\
\text { gener Atmungsstö- } \\
\text { rungen } \\
\text { - Vertiefte Grundla- } \\
\text { gen: Therapiemög- } \\
\text { lichkeiten der } \\
\text { Schlafapnoe }\end{array}$ & $\begin{array}{l}\text { - Aktueller Lebenslauf } \\
\text { - Facharzturkunde } \\
\text { Innere Medizin und } \\
\text { Kardiologie bzw. } \\
\text { Innere Medizin } \\
\text { mit Schwerpunkt } \\
\text { Kardiologie } \\
\text { - Logbuch } \\
\text { - Detailliertes Zeugnis } \\
\text { des Leiters der Zu- } \\
\text { satzqualifikation mit } \\
\text { Angabe der Qualifi- } \\
\text { zierungszeiträume } \\
\text { der verschiedenen } \\
\text { Stufen (mit durch- } \\
\text { geführten Prozedu- } \\
\text { ren), der Aktivitäten } \\
\text { und erworbenen } \\
\text { Kompetenz und } \\
\text { erreichten Selbst- } \\
\text { ständigkeit } \\
\text { - Nachweis der } \\
\text { CME-Punkte }\end{array}$ \\
\hline
\end{tabular}




\begin{tabular}{|c|c|c|c|c|}
\hline Stufe & Voraussetzungen & Mindestzahlen & Qualifizierungsinhalt & $\begin{array}{l}\text { Einzureichende Un- } \\
\text { terlagen }\end{array}$ \\
\hline 3 & $\begin{array}{l}\text { Strukturierte Qualifizierung im } \\
\text { Rahmen von Vorträgen im Rah- } \\
\text { men der Weiterbildungsakademie: } \\
30 \text { CME-Punkte oder Analog } \\
\text { BUB-Kurs } \\
6 \text { Monate in Vollzeit an einer von } \\
\text { der DGK nach Curriculum Kardio- } \\
\text { vaskuläre Schlafmedizin zertifi- } \\
\text { zierten Stätte, davon mindestens } \\
3 \text { Monate in Vollzeit im Schlaflabor }\end{array}$ & $\begin{array}{l}\text { Logbuch-Nachweis: } \\
\text { Mindestens } 150 \text { schlafmedizinische Untersuchun- } \\
\text { gen, davon: } \\
\text { - mindestens } 100 \text { diagnostische Polysomnogra- } \\
\text { phien } \\
\text { - mindestens } 50 \text { Polysomnographien zur Einlei- } \\
\text { tung einer Positivdrucktherapie } \\
\text { - selbstständige Befundung aller Untersuchun- } \\
\text { gen } \\
\text { Mentor: Facharzt für Innere Medizin und Kar- } \\
\text { diologie bzw. Innere Medizin mit Schwerpunkt } \\
\text { Kardiologie mit persönlicher Anerkennung der } \\
\text { Stufe } 3 \text { der Zusatzqualifikation } \\
\text { Optional gilt folgende Regelung: Leiter des ko- } \\
\text { operierenden Schlaflabors mit detaillierten } \\
\text { Kenntnissen in der kardiovaskulären Schlafme- } \\
\text { dizin (s. Abschn. 7) } \\
\text { Maximaldauer der Programmteilnahme: } \\
24 \text { Monate }\end{array}$ & $\begin{array}{l}\text { - Fundierte Kenntnis- } \\
\text { se der Pathophysio- } \\
\text { logie schlafbezo- } \\
\text { gener Atmungsstö- } \\
\text { rungen } \\
\text { - Fundierte Kennt- } \\
\text { nisse in der Durch- } \\
\text { führung und Inter- } \\
\text { pretation der kar- } \\
\text { diorespiratorischen } \\
\text { Polygraphie und } \\
\text { Polysomnographie, } \\
\text { aber auch detail- } \\
\text { lierte Kenntnisse } \\
\text { und Erfahrungen } \\
\text { in den zur Verfü- } \\
\text { gung stehenden } \\
\text { Therapieoptionen } \\
\text { - Selbstständige } \\
\text { Diagnostik und Dif- } \\
\text { ferenzialdiagnostik } \\
\text { schlafbezogener } \\
\text { Atmungsstörungen } \\
\text { - Selbstständige The- } \\
\text { rapie von schlafbe- } \\
\text { zogenen Atmungs- } \\
\text { störungen }\end{array}$ & $\begin{array}{l}\text { - Aktueller Lebenslauf } \\
\text { - Facharzturkunde } \\
\text { Innere Medizin und } \\
\text { Kardiologie bzw. } \\
\text { Innere Medizin } \\
\text { mit Schwerpunkt } \\
\text { Kardiologie } \\
\text { - Logbuch } \\
\text { - Detailliertes Zeugnis } \\
\text { des Leiters der Zu- } \\
\text { satzqualifikation mit } \\
\text { Angabe der Qualif- } \\
\text { zierungszeiträume } \\
\text { der verschiedenen } \\
\text { Stufen (mit durch- } \\
\text { geführten Prozedu- } \\
\text { ren), der Aktivitäten } \\
\text { und erworbenen } \\
\text { Kompetenz und } \\
\text { erreichten Selbst- } \\
\text { ständigkeit } \\
\text { - Nachweis der } \\
\text { CME-Punkte }\end{array}$ \\
\hline
\end{tabular}

Tab. 3 Rezertifizierung der persönlichen Anerkennung der Zusatzqualifikation Kardiovaskuläre Schlafmedizin - Übersicht der Voraussetzungen und Kriterien für die 3 Stufen der Qualifizierung

\begin{tabular}{|l|l|l|l|}
\hline Stufe & $\begin{array}{l}\text { Voraus- } \\
\text { setzungen }\end{array}$ & Zahl der Patientenuntersuchungen & Einzureichende Unterlagen \\
\hline 1 & $\begin{array}{l}\text { Anerkennung } \\
\text { der Stufe 1 }\end{array}$ & $\begin{array}{l}30 \text { schlafmedizinische Untersuchungen } \\
\text { (PG/PSG) pro Jahr der Zertifizierungsdauer }\end{array}$ & $\begin{array}{l}\text { - Nachweis der erforderlichen Patientenuntersuchungen durch Kopien der } \\
\text { Untersuchungsbefunde in anonymisierter Form } \\
-\begin{array}{l}\text { Nachweis über 2 CME-Punkte pro Jahr der Gültigkeit des auslaufenden } \\
\text { Zertifikats }\end{array}\end{array}$ \\
\hline 2 & $\begin{array}{l}\text { Anerkennung } \\
\text { der Stufe 2 }\end{array}$ & $\begin{array}{l}50 \text { schlafmedizinische Untersuchungen } \\
\text { (PG/PSG) pro Jahr der Zertifizierungsdauer }\end{array}$ & $\begin{array}{l}\text { - Nachweis der erforderlichen Patientenuntersuchungen durch Kopien der } \\
\text { Untersuchungsbefunde in anonymisierter Form } \\
- \text { Nachweis über 6 CME-Punkte pro Jahr der Gültigkeit des auslaufenden } \\
\text { Zertifikats }\end{array}$ \\
\hline 3 & $\begin{array}{l}\text { Anerkennung } \\
\text { der Stufe 3 }\end{array}$ & $\begin{array}{l}100 \text { schlafmedizinische Untersuchungen } \\
\text { als primärer Befunder (PG/PSG) pro Jahr } \\
\text { der Zertifizierungsdauer }\end{array}$ & $\begin{array}{l}\text { Nachweis der entsprechenden Zahlen durch Kopien der Untersuchungsbe- } \\
\text { funde in anonymisierter Form } \\
- \text { Nachweis über 12 CME-Punkte pro Jahr der Gültigkeit des auslaufenden } \\
\text { Zertifikats }\end{array}$ \\
\hline
\end{tabular}

- Ablauf der Messungen: Mit Vor- und Nachbereitung der Messungen (Polysomnographie oder Polygraphie) ist ein Zeitraum vorzusehen, der einen mindestens 6-stündigen Schlaf- und Überwachungszeitraum pro Patient - unter Berücksichtigung individueller Bett- und Schlafzeiten - gewährleistet.

- Für die unmittelbare Vor- und Nachbereitung einer Polysomnographie sind mindestens 45 bis 60 min anzusetzen.

- Die Stätte muss regelmäßig (mindestens 2-mal/Jahr) schlafmedizinische
Fortbildungen für die Mitarbeiter durchführen.

- Als apparative Grundausstattung sind mindestens 1 Polysomnographieeinheit sowie mindestens 1 Polygraphieeinheit gefordert.

- Zu den apparativen Voraussetzungen der Polysomnographie zählen Elektroenzephalogramm(EEG)-Ableitungen, Elektrookulogramm(EOG)Ableitungen, Elektromyogramm (EMG) mentalis oder submentalis, getrennte Aufzeichnung der Atmungsbewe- gungen an Thorax und Abdomen, oronasaler Luftfluss, Sauerstoffsättigung, Schnarchen, Lage, eine EKGAbleitung, mindestens 1 M.-tibialisEMG.

- Die Ableittechnik (Polysomnographie) muss die Voraussetzungen für Ableitungen nach dem jeweils aktuellen Standard der American Academy of Sleep Medicine (AASM) erfüllen. Alle heute erhältlichen Polysomnographen erlauben eine Signalkonfiguration nach AASM. 
Tab. 4 Anerkennung als Stätte der Zusatzqualifikation Kardiovaskuläre Schlafmedizin - Übersicht der Voraussetzungen und Kriterien

\begin{tabular}{|c|c|c|}
\hline Voraussetzungen & Mindestzahlen & Einzureichende Unterlagen \\
\hline $\begin{array}{l}\text { Möglichkeit sowohl der kardiologischen Diagnostik und Therapie als auch der Polygraphie, Po- } \\
\text { lysomnographie und Therapieeinleitung bzw. -kontrolle schlafbezogener Atmungsstörungen }\end{array}$ & \multirow{5}{*}{$\begin{array}{l}\text { - Mindestens } \\
250 \text { Poly- } \\
\text { graphien } \\
\text { und/oder } \\
\text { Polysom- } \\
\text { nographien } \\
\text { pro Jahr } \\
\text { - Mindestens } \\
100 \text { Thera- } \\
\text { pieeinleitun- } \\
\text { gen und 100 } \\
\text { Therapien- } \\
\text { achsorgen } \\
\text { pro Jahr }\end{array}$} & \multirow{5}{*}{$\begin{array}{l}\text { - Ausführliche Beschreibung } \\
\text { der Stätte, die Aufschluss } \\
\text { über die personelle, räumli- } \\
\text { che und apparative Ausstat- } \\
\text { tung sowie den Ablauf und } \\
\text { die Struktur in der Stätte } \\
\text { gibt und Angaben zu wö- } \\
\text { chentlichen Konferenzen } \\
\text { und internen (evtl. auch } \\
\text { externen) Fortbildungen } \\
\text { enthält } \\
\text { - Nachweisdokumente der } \\
\text { gesetzlich geregelten Qua- } \\
\text { litätsvorgaben }\end{array}$} \\
\hline $\begin{array}{l}\text { Personelle Ausstattung: } \\
\text { - Stufe-3-zertifizierter Leiter der Zusatzqualifikation } \\
\text { - Optional Stufe-3-zertifizierter stellv. Leiter der Zusatzqualifikation } \\
\text { - MTA/MFA/Pfleger mit Erfahrung in PSG/Therapieeinleitung im Tag- sowie Nachtdienst }\end{array}$ & & \\
\hline $\begin{array}{l}\text { Räumliche Ausstattung: } \\
\text { - Separates Schlaflabor, kann räumlich getrennt vom Krankenhaus sein } \\
\text { - Möglichkeit der Durchführung der Polysomnographie im Einzelzimmer } \\
\text { - Möglichkeit zur Device-Abfrage }\end{array}$ & & \\
\hline $\begin{array}{l}\text { Apparative Ausstattung: } \\
->1 \text { Polysomnographieeinheit und }>1 \text { Polygraphieeinheit } \\
\text { - Archivsystem } \\
\text { - Gegensprechanlage }\end{array}$ & & \\
\hline $\begin{array}{l}\text { Konferenzen/Fortbildungen: } \\
\text { - Fallkonferenzen mit dem (stellv.) Leiter der Stätte (wöchentlich) } \\
\text { - Die Stätte muss regelmäßig (mindestens 2-mal/Jahr) schlafmedizinische Fortbildungen für die } \\
\text { Mitarbeiter durchführen }\end{array}$ & & \\
\hline
\end{tabular}

Tab. 5 Anerkennung als (stellv.) Leiter der Zusatzqualifikation Kardiovaskuläre Schlafmedizin - Übersicht der Voraussetzungen und Kriterien

\begin{tabular}{|c|c|c|c|}
\hline \multicolumn{2}{|c|}{ Voraussetzungen } & Mindestzahlen & Einzureichende Unterlagen \\
\hline \multirow[t]{4}{*}{1.} & $\begin{array}{l}\text { Facharztanerkennung Innere Medizin und Kardiologie bzw. Innere } \\
\text { Medizin mit Schwerpunkt Kardiologie }\end{array}$ & \multirow{8}{*}{$\begin{array}{l}\text { In den letzten } 5 \text { Jahren vor Antrag- } \\
\text { stellung Initiierung und Interpreta- } \\
\text { tion von } \\
\text { - mindestens } 50 \text { vereinfachten } \\
\text { apparativen diagnostischen } \\
\text { Verfahren zur Stellung der Ver- } \\
\text { dachtsdiagnose schlafbezogene } \\
\text { Atmungsstörung pro Jahr (z. B. } \\
\text { 1- bis 2-Kanal-Aufzeichnungen } \\
\text { und/oder Aufzeichnungen aus } \\
\text { implantierbaren elektrischen } \\
\text { Devices) und } \\
\text { - } 50 \text { kardiorespiratorischen Poly- } \\
\text { graphien pro Jahr sowie } \\
\text { - Verantwortung von mindestens } \\
50 \text { Therapieeinleitungen pro } \\
\text { Jahr und } \\
\text { - } 50 \text { Therapiekontrollen pro Jahr }\end{array}$} & \multirow{8}{*}{$\begin{array}{l}\text { - Aktueller Lebenslauf } \\
\text { - Facharzturkunde Innere Me- } \\
\text { dizin und Kardiologie bzw. } \\
\text { Innere Medizin mit Schwer- } \\
\text { punkt Kardiologie } \\
\text { - Zertifikat der persönlichen } \\
\text { Stufe-3-Anerkennung } \\
\text { - Zeugnis des Vorgesetzten } \\
\text { mit Angaben zu Kenntnissen } \\
\text { im Bereich kardiovaskulä- } \\
\text { re Schlafmedizin, Untersu- } \\
\text { chungszahlen und Beschäfti- } \\
\text { gungsumfang }\end{array}$} \\
\hline & Detaillierte Kenntnisse im Bereich kardiovaskuläre Schlafmedizin & & \\
\hline & Persönliche Anerkennung der Zusatzqualifikation Stufe 3 & & \\
\hline & Vollzeittätigkeit an der Stätte der Zusatzqualifikation & & \\
\hline \multirow[t]{4}{*}{2.} & $\begin{array}{l}\text { Optional: Leiter des kooperierenden Schlaflabors und Leiter der Zu- } \\
\text { satzqualifikation: } \\
\text { Facharztanerkennung für Allgemeinmedizin, Hals-Nasen-Ohren-Heil- } \\
\text { kunde, Innere Medizin, Innere Medizin und PneumologieAQ13, } \\
\text { Innere Medizin in Verbindung mit der Schwerpunktbezeichnung } \\
\text { Pneumologie, Neurologie, Nervenheilkunde oder Psychiatrie und } \\
\text { Psychotherapie }\end{array}$ & & \\
\hline & Detaillierte Kenntnisse im Bereich kardiovaskuläre Schlafmedizin & & \\
\hline & Vollzeittätigkeit an der Stätte der Zusatzqualifikation & & \\
\hline & $\begin{array}{l}\text { Bei 2. (optional) muss der stellv. Leiter der Zusatzqualifikation die } \\
\text { Voraussetzungen unter 1. erfüllen }\end{array}$ & & \\
\hline
\end{tabular}

Für die Rezertifizierung des (stellv.) Leiters der Zusatzqualifikation gelten die gleichen Bedingungen wie für die Erstzertifizierung

- Ein Archivsystem für alte Polysomnographien muss den Zugriff auf zurückliegende Befunde und Polysomnographien ermöglichen.

- Eine Gegensprechanlage gewährleistet die Verständigung zwischen Patient und Nachtwache. Sie kann außerdem zur Biosignaleichung und Audioüberwachung eingesetzt werden.

Mit dem Antrag auf Anerkennung als (stellv.) Leiter der Zusatzqualifikation sind ein aktueller Lebenslauf, die Facharzturkunde Kardiologie, der Nachweis über die persönliche Stufe-3-Anerkennung der Zusatzqualifikation sowie ein Zeugnis des Leiters der jeweiligen Einrichtung über die für (stellv.) Leiter der Zusatzqualifikation geforderten Mindestzahlen einzureichen. Die Anerkennung als Stätte der Zusatzqualifikation wird befristet erteilt. Danach muss für die Fortdauer der Anerkennung eine Rezertifizierung erfolgen.

Für strittige Fälle wird eine Schiedsstelle bei der DGK eingerichtet.

\section{9. Übergangsregelung}

Fachärzte für Innere Medizin und Kardiologie bzw. Innere Medizin mit Schwerpunkt Kardiologie, die in mindestens 2 der letzten 5 Jahre vor Antragstellung überwiegend auf dem Gebiet der Schlaf- und Beatmungsmedizin tätig waren, können auch ohne formales Durchlaufen des Curriculums die Zusatzqualifikation Kardiovaskuläre Schlafmedizin für Stufe 3 erwerben. Die überwiegende Tätigkeit im Bereich Schlaf- und Beatmungsmedizin sowie die geforderten Mindestzahlen müs- 
Hier steht eine Anzeige.

黑 Springer 
Hier steht eine Anzeige.

黑 Springer 
sen durch den Leiter der jeweiligen Einrichtung bestätigt werden. Außerdem benötigen die Kandidaten der Übergangsregelung eine Bestätigung der erfolgreichen Teilnahme an einem BUB-Kurs oder alternativ die gültige Anerkennung durch die KV zur Abrechnung schlafmedizinischer Leistungen.

Die von den Kandidaten der Übergangsregelung zu erfüllenden Kriterien und einzureichenden Dokumente und Nachweise sind in $\bullet$ Tab. 1 unter Abschn. 12 zusammengefasst.

Die Übergangsregelung gilt für 2 Jahre ab Publikation dieses Curriculums. Nach Ablauf dieser Frist ist die Erlangung der Zusatzqualifikation Kardiovaskuläre Schlafmedizin nur noch durch Teilnahme an dem entsprechenden Qualifizierungsprogramm möglich.

\section{Rezertifizierung}

Die Gültigkeit der Anerkennung sowohl von Personen als auch von Stätten und deren (stellv.) Leitern der Zusatzqualifikation ist befristet und unterliegt somit einer Rezertifizierungspflicht, sofern die jeweilige Anerkennung weiterbestehen soll.

Die Rezertifizierung von Personen dient dem Zweck des Nachweises, dass die zertifizierte Person nach wie vor im Fachgebiet der kardiovaskulären Schlafmedizin tätig ist.

Die Rezertifizierung einer Stätte und ihres (stellv.) Leiters der Zusatzqualifikation ist durch den aktuellen Leiter der Zusatzqualifikation zu beantragen und dient dem Zweck des Nachweises, dass die im Zuge der Erstzertifizierung genannten personellen, apparativen und logistischen Voraussetzungen weiterhin erfüllt sind.

Die für die persönliche Rezertifizierung zu erfüllenden Kriterien und einzureichenden Dokumente und Nachweise für die 3 Qualifizierungsstufen sind in - Tab. 3 unter Abschn. 12 zusammengefasst. Für die Rezertifizierung einer Stätte und ihres (stellv.) Leiters der Zusatzqualifikation gelten die gleichen Voraussetzungen wie bei der Erstzertifizierung. Darüber hinaus ist für die Rezertifizierung der Stätte der Nachweis erforderlich, dass die Stätte im laufenden Zertifizierungszeitraum aktiv Programmkandidaten ausgebildet hat.

\section{Curriculum cardiovascular sleep medicine}

Sleep-related breathing disorders are very common in cardiovascular disease patients with, for example, difficult-to-control arterial hypertension, coronary artery disease, valvular heart disease, cardiac arrhythmias, or heart failure, and are associated with a worse prognosis. The therapy of sleep-related breathing disorders can usefully complement the treatment of cardiovascular diseases because of its effects on arterial blood pressure and quality of life in selected patients. This makes integrative cardiology and sleep medicine care for these patients all the more important. Therefore, the purpose of the curriculum is to describe a qualification process, the goal of which is to obtain the additional qualification Cardiovascular Sleep Medicine. This is intended to improve the qualification in this specialized field so that it exceeds the level of general residency training in cardiology. The curriculum has a three-tiered structure. Level 1 systematically imparts basic knowledge and skills according to the qualification contents of the curriculum cardiology, level 2 imparts knowledge and skills, among others, for the independent performance of polygraphy and level 3, among others, for the independent performance of polysomnography.

\section{Keywords}

Obstructive sleep apnea · Polygraphy · Polysomnography · Quality · Qualification

\section{Qualitätskontrolle}

Die Stätten der Zusatzqualifikation stellen den vom Vorstand der DGK benannten Gutachtern die Nachweisdokumente der gesetzlich geregelten Qualitätsvorgaben zur Verfügung.

\section{2. Übersicht der erforderlichen Voraussetzungen}

(•Tab. 1, 2, 3, 4 und 5)

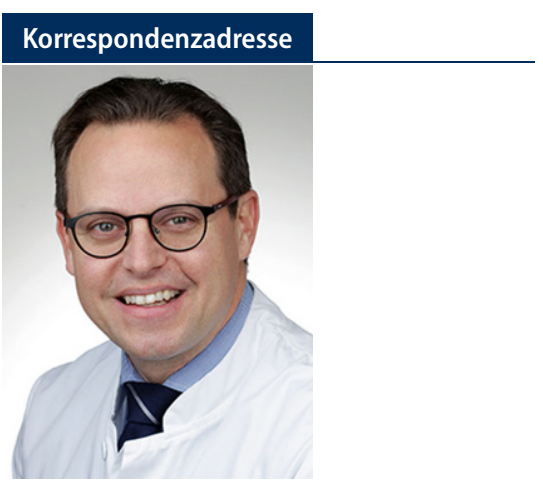

Prof. Dr. M. Arzt

Klinik und Poliklinik für Innere Medizin II, Universitätsklinikum Regensburg Franz-Josef-Strauß-Allee 11, 93053 Regensburg, Deutschland michael.arzt@ukr.de

\section{Einhaltung ethischer Richtlinien}

Interessenkonflikt. Den Interessenkonflikt der Autoren finden Sie online auf der DGK-Homepage unter http://leitlinien.dgk.org/ bei der entsprechenden Publikation.

Für diesen Beitrag wurden von den Autoren keine Studien an Menschen oder Tieren durchgeführt. Für die aufgeführten Studien gelten die jeweils dort angegebenen ethischen Richtlinien.

\section{Literatur}

1. Arzt M, Woehrle H, Oldenburg $O$ et al (2016) Prevalence and predictors of sleep-disordered breathing in patients with stable chronic heart failure: The SchlaHF registry. JACC Heart Fail 4:116-125

2. Bitter T, Westerheide N, Prinz C et al (2011) Cheyne-Stokes respiration and obstructive sleep apnoea are independent risk factors for malignant ventricular arrhythmias requiring appropriate cardioverter-defibrillator therapies in patients with congestive heart failure. Eur Heart J 32:61-74

3. Buchner S, Satzl A, Debl K et al (2014) Impact of sleep-disordered breathing on myocardial salvage and infarct size in patients with acute myocardial infarction. Eur Heart J 35:192-199

4. Gami AS, Hodge DO, Herges RM et al (2007) Obstructive sleep apnea, obesity, and the risk of incident atrial fibrillation. J Am Coll Cardiol 49:565-571

5. Gami AS, Howard DE, Olson EJ et al (2005) Daynight pattern of sudden death in obstructive sleep apnea. NEngl J Med 352:1206-1214

6. Gami AS, Olson EJ, Shen WKetal (2013) Obstructive sleep apnea and the risk of sudden cardiac death: a longitudinal study of 10,701 adults. J Am Coll Cardiol 62:610-616

7. Gottlieb DJ, Yenokyan G, Newman AB et al (2010) Prospective study of obstructive sleep apnea and incident coronary heart disease and heart 


\section{Curriculum}

failure: the sleep heart health study. Circulation 122:352-360

8. Hombach A, Kelle S, Gebker R et al (2014) Curriculum Kardiale Magnetresonanztomographie (CMR). Kardiologe 8:451-461

9. Khayat R, Jarjoura D, Porter K et al (2015) Sleep disordered breathing and post-discharge mortality in patients with acute heart failure. Eur Heart J36:1463-1469

10. LinzD, WoehrleH,BitterTetal(2015) The importance of sleep-disordered breathing in cardiovascular disease. Clin Res Cardiol 104:705-718

11. Ng CY, Liu T, Shehata M et al (2011) Meta-analysis of obstructive sleep apnea as predictor of atrial fibrillation recurrence after catheter ablation. Am J Cardiol 108:47-51

12. Oldenburg $\mathrm{O}$, Wellmann B, Buchholz A et al (2016) Nocturnal hypoxaemia is associated with increased mortality in stable heart failure patients. EurHeart J37:1695-1703

13. Schädlich S, Warmuth R, Rodenbeck A et al (2017) Leitfaden und Kriterien für die Akkreditierung von Schlaflaboren der Deutschen Gesellschaft für Schlafforschung und Schlafmedizin (DGSM). Somnologie 21:200-209

14. WerdanK,EckardtL, Elsässer Aetal (2015) Erteilung einer Weiterbildungsbefugnis für die Facharztkompetenz "Innere Medizin und Kardiologie": Empfehlungen zur Antragstellung. Kardiologe 9:354-362

15. Werdan K, Flachskampf F, Griebenow R et al (2013) Curriculum Kardiologie. Kardiologe 7:435-456

16. Yumino D, Wang $H$, Floras JS et al (2009) Relationship between sleep apnoea and mortality in patients with ischaemic heart failure. Heart 95:819-824

\section{Dem Geheimnnis des EKGs auf der Spur: 108 Fälle im Intensivkurs EKG}

Bei einem 73-jährigen Patienten bestehen Schwindel und Dyspnoe unter Belastung. Dieses EKG liegt Ihnen zur Befundung vor.

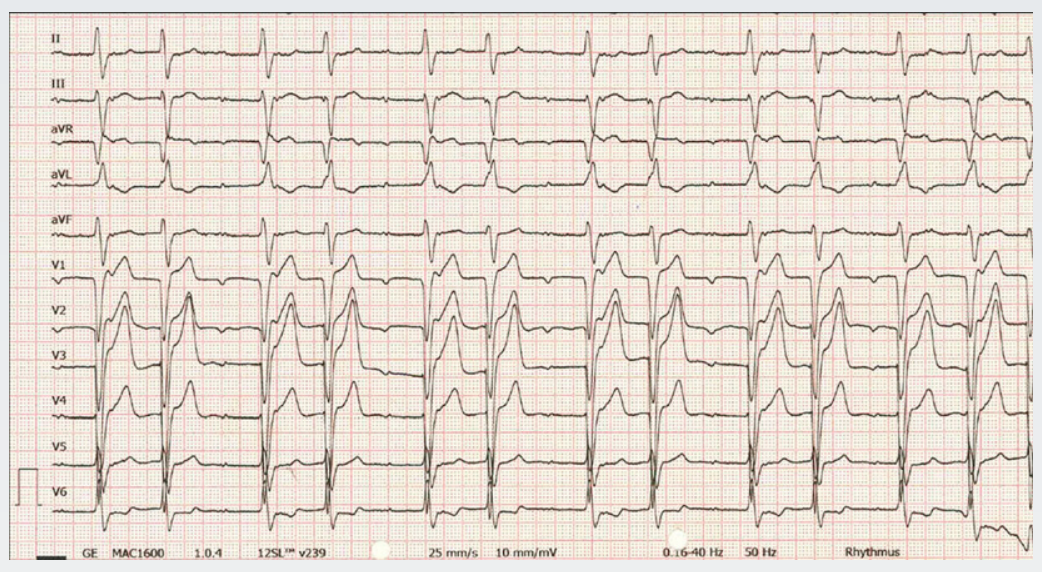

Was meinen Sie?

a) Es besteht Vorhofflattern mit unregelmäßiger Überleitung.

b) Es besteht ein AV-Block $I^{\circ}$ und ein unbestimmter Schenkelblock.

c) Es besteht ein AV-Block $\|^{\circ}$ Typ Wenckebach (= Mobitz 1).

Die Lösung dieses Falls sowie weitere spannende 107 EKG-Fälle in unterschiedlichen Schwierigkeitsstufen mit Lösungshinweisen und ausführlichen Kommentaren finden Sie im Intensivkurs-EKG von Herrn PD Dr. Carsten W. Israel, Bielefeld. Verbessern Sie Ihre Routine in der Interpretation von EKG-Aufzeichnungen!

Einfach diesen QR-Code scannen:

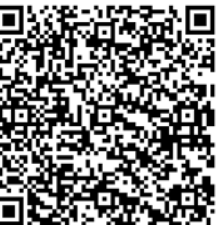

